\title{
„Studie lässt nur eingeschränkte Aussagen zu“
}

\begin{abstract}
Herr Professor Ihl, die Autoren der GuidAge-Studie haben ein negatives Fazit gezogen, was die Möglichkeit betrifft, mit Ginkgo biloba der Entstehung eines Morbus Alzheimer vorzubeugen. Sie haben selbst viel über die Substanz geforscht. Sind Sie enttäuscht? Professor Ralf Ihl: Nein. Die Rahmenbedingungen der Studie waren nicht geeignet, eine Aussage zur präventiven Wirksamkeit zuzulassen, was die Autoren selbst beschreiben und übrigens auch in einem Kommentar von Lon Schneider im „Lancet Neurology" anklingt. Leider widersprechen sie sich dann selbst und ziehen ohne hinreichende Datenlage negative Schlüsse.
\end{abstract}

Vor allem in der Post-hoc-Analyse räumen die Autoren aber zumindest die Möglichkeit eines präventiven Effekts nach mehr als dreijähriger Ginkgo-Einnahme ein. Halten Sie das für realistisch?

Ihl: Die Autoren machen auf einen wichtigen Aspekt aufmerksam, den schon frühere Studien gezeigt haben: Wenn man wissen will, ob eine Substanz präventiv wirkt, muss sie auch eingenommen werden. Interessanterweise war eine solche Vorgehensweise im Studiendesign vorgesehen und durchgeführt worden. Sie erbrachte einen positiven Effekt. Gesetzt den Fall, dass diese

\section{Studie "GuidAge" in Kürze}

Ein Extrakt von Ginkgo biloba hat in der Verumgruppe der GuidAgeStudie die Inzidenz von Morbus Alzheimer nicht signifikant gesenkt. Dennoch könnte es sein, dass die langzeitige Einnahme protektiv wirkt.

An der multizentrischen, randomisierten, doppelblinden und placebokontrollierten Studie hatten sich über 2.800 Frauen und Männer in mehr als 700 Allgemeinarztpraxen beteiligt. Sie waren alle über 70 Jahre alt und hatten bei ihrem Hausarzt über Gedächtnisprobleme geklagt. Jeweils rund 1.400 Studienteilnehmer erhielten daraufhin $2 \times 120 \mathrm{mg} / \mathrm{d}$ standardisierten Ginkgo-biloba-Extrakt (EGb761) oder Placebo. Fünf Jahre lang wurden die Probanden von ihren Hausärzten und in spezialisierten Gedächtniszentren nachbeobachtet. Am Studienende hatten knapp $5 \%$ eine Demenz entwickelt - statt, wie vorausberechnet, rund $14 \%$. Die statistische Aussagekraft der Studie wurde dadurch geschwächt. Die Alzheimer-Inzidenzen in der Placebo- (5,2\%) und der Verumgruppe $(4,3 \%)$ waren statistisch nicht signifikant verschieden $(p=0,3)$. Eine Post-hoc-Analyse ergab in der Ginkgo-Gruppe allerdings nahezu eine Halbierung des Risikos, an Alzheimer zu erkranken - vorausgesetzt, der Extrakt wurde länger als drei Jahre eingenommen $(p=0,02)$. Für Demenzen allgemein betrug die Reduktion $47 \%$, ebenfalls unter der Maßgabe langzeitiger Einnahme $(p=0,02)$. Es könne sich bei den Ergebnissen um Zufall handeln, schreiben die Forscher und verweisen auf die geringe Gesamtzahl von Alzheimer-Erkrankungen in der Studie. Allerdings habe bereits eine frühere Studie zur Alzheimer-Prävention mit nicht-steroidalen Antirheumatika darauf hingedeutet, dass sich eine vorbeugende Behandlung im Zeitverlauf unterschiedlich auswirken könnte [Breitner JC et al. Alzheimers Dement 2011; 7: 402-11].

Vellas B et al. Long-term use of standardised ginkgo biloba extract for the prevention of Alzheimer's disease (GuidAge): a randomized placebo-controlled trial. Lancet Neurol 2012, online 6. September; http:// dx.doi.org/10.1016/S1474-4422(12)70206-5

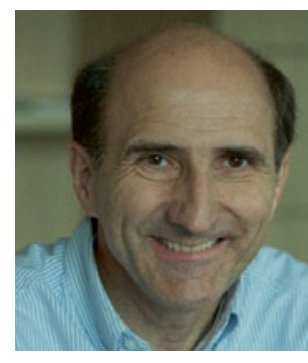

Prof. Dr. med. Ralf Ihl

Chefarzt Gerontopsychiatrie und -psychotherapie der Alexianer Krefeld $\mathrm{GmbH}$

Größenordnung tatsächlich zutrifft, was aufgrund der Daten der Vorstudien EPIDOS, GEM und PAQUID nicht ausgeschlossen ist, wäre es dringend notwendig, die Daten weiterzuverfolgen.

\section{Was ist an der Studie kritikwürdig?}

Ihl: Schneider bezeichnet in seinem Kommentar Präventionsstudien zu Recht als „Pionierarbeit“. Da bleiben Fehler nicht aus. Wir gehen heute davon aus, dass Demenzen schon 20 bis 30 Jahre vor der Diagnose entstehen. Es steht damit infrage, ob es günstig ist, eine Studie in einer Population mit so hohem Durchschnittsalter durchzuführen und auch nur über so kurze Zeit. Hier ist das Risiko sehr hoch, dass man bei bereits erkrankten Menschen eine Vorbeugung versucht. Das kann naturgemäß eher frustran sein.

Auffällig ist die - im Vergleich zur eingangs der Studie angenommenen - insgesamt niedrige Demenzinzidenz in der Studienpopulation. Wie ist das mit Blick auf die Ergebnisse zu beurteilen?

Ihl: Wenn man die Hypothese einer Wirksamkeit aufgestellt hat, lässt sie sich deutlich schlechter bestätigen, wenn die geschätzte Konversionsrate, wie hier geschehen, deutlich verfehlt wird. Positive Effekte können damit sehr leicht übersehen werden.

Wirkt Ginkgo nun präventiv - und wenn ja, welche Patienten profitieren davon am meisten?

Ihl: Die GuidAge-Studie lässt nur äußerst eingeschränkte Aussagen zu. Legt man die Subanalyse zugrunde, was alleine nicht hinreichend ist, kann man sie als Hinweis auf einen wichtigen Umstand werten, der bereits in der GEM-Studie beschrieben wurde und eine wesentliche Erkenntnis der EPIDOS-Studie war: Wenn die Substanz eingenommen wird, könnte sie, wenn die Einnahme hinreichend lange erfolgt, einen relevanten präventiven Einfluss haben. Um diese Aussage zu festigen, wären weitere Daten nötig. In eine geeignete Studie wären jüngere Probanden aufzunehmen, weil man vermutlich ab Mitte 40 bis 50 mit der Prävention beginnen müsste. Die Teilnehmerzahl müsste größer sein und die Untersuchung etwa 20 Jahre dauern. Es ist fraglich, ob eine solch teure Studie einen Investor finden würde. Billiger wäre es zu prüfen, ob Teilnehmer aus früheren Studien wie EPIDOS oder PAQUID noch erreichbar sind. So könnten recht rasch Langzeitdaten zur Verfügung stehen. 